\title{
Gestational age, not transient hyperthyrotropinemia impacts brain white matter diffusion tensor imaging in premature infants
}

\author{
PI-LIEN HUNG ${ }^{1}$, CHUN-CHUNG LUI ${ }^{2}$, CHEN-CHANG LEE ${ }^{2}$, YIN-HSIU CHIEN ${ }^{3}$, FENG-SHUN CHEN ${ }^{1}$, \\ CHIH-CHENG CHEN ${ }^{1}$, HONG-REN YU ${ }^{1}$, MEI-YUNG CHUNG ${ }^{1}$ and LI-TUNG HUANG ${ }^{1}$ \\ Departments of ${ }^{1}$ Pediatrics and ${ }^{2}$ Radiology, Kaohsiung Chang Gung Memorial Hospital and \\ Chang Gung University College of Medicine, Kaohsiung 83301; ${ }^{3}$ Department of Medical Genetics, \\ National Taiwan University Hospital, Taipei 10048, Taiwan, R.O.C.
}

Received June 30, 2017; Accepted November 2, 2017

DOI: $10.3892 / \mathrm{etm} .2017 .5440$

\begin{abstract}
Transient hypothyroidism is common in premature infants and increases the risk of adverse neurodevelopmental outcomes. Thyroid hormone (TH) is involved in oligodendrocyte development and myelination, however, whether transient hypothyroidism is associated with oligodendrocyte dysplasia and abnormal myelination is unclear. The aim of the present study was to investigate correlations among $\mathrm{TH}$ levels, neurodevelopmental outcomes and white matter (WM) microstructure in premature infants. The authors designed a cohort study recruiting 81 premature infants (age, 23-35 weeks). A total of 17 were born with a gestational age $(\mathrm{GA})<30$ weeks (early preterm group) and 64 of them were born with a GA $\geq 30$ weeks (late preterm group). For outcome measurement, thyroid stimulating hormone (TSH) levels at 0,18 , and $24 \mathrm{~h}$ of admission were measured. Neurodevelopmental outcomes were assessed using Bayley III test. Diffusion tensor imaging was used to explore the characterization of WM microstructure. The data demonstrated that GA, however not TSH level was associated with neurodevelopmental outcomes in the following 2 years. Fractional anisotrophy (FA) increased with TSH0 levels over anterior limb of internal capsule, while axial diffusivity decreased with TSH0 levels over splenium of corpus callosum (CC). The late preterm group had more intact WM integrity over the internal and external capsule (EC) in FA compared with the early preterm group. Infants with motor dysfunction had significantly increased mean diffusivity (MD) values at regions of interest in the genu and splenium of $\mathrm{CC}$.
\end{abstract}

Correspondence to: Dr Li-Tung Huang, Department of Pediatrics, Kaohsiung Chang Gung Memorial Hospital and Chang Gung University College of Medicine, 123 Ta-Pei Road, Niao-Song, Kaohsiung 83301, Taiwan, R.O.C.

E-mail: huangli@cgmh.org.tw

Key words: transient hypothyroidism in premature infants, thyroid stimulating hormone, performance developmental index, diffusion tensor imaging, mean diffusivity
The results of the present study demonstrated that GA, however not transient hypothyroidism influenced neurodevelopmental outcomes in the premature infants. FA increased with age in a regionally-specific manner over regions of the internal capsule and EC. MD may act as a potential predictor for motor function in premature babies.

\section{Introduction}

Thyroid hormones (TH) are critical for brain development throughout gestation and the first year after birth (1). However, thyroid dysfunction is common in preterm infants, and this has been associated with neurodevelopmental delays and cognitive dysfunction in later life (2-5). Transient hyperthyrotropinemia (HTT) with hypothyroxinemia is common in preterm infants. Initial HTT can lead to transient self-limited hypothyroidism or permanent hypothyroidism requiring $\mathrm{TH}$ replacement therapy. Preterm infants are at significant risk of congenital hypothyroidism, which is unpredictable in its evolution to transient or permanent (6). Several physiologic and non-physiologic factors may contribute to hypothyroidism in premature infants, including immaturity of the hypothalamic-pituitary-thyroid axis, limited thyroid capacity to concentrate and synthesize iodine, increase in $\mathrm{TH}$ requirement needs for thermogenesis, iodine insufficiency, and preterm diseases (7). TH play an important role in myelin development, in the production and maturation of oligodendrocytes, and on the expression of genes encoding myelin (8). The period of TH sensitivity with regards the myelin gene has been reported to extend from about the end of the first postnatal week in rat brains, which is equivalent to late gestation in human brains, which is the high-risk period of periventricular leukomalacia, a major cause of cerebral palsy (CP) (9). TH deficiency may impair myelination by alternating the timing of the differentiation of oligodendrocyte precursor cells and interrupts the proliferation of precursor cells. The commonest magnetic resonance imaging (MRI) finding in children with $\mathrm{CP}$ is volume reduction of periventricular white matter (WM), which provided the evidence of myelination impairment (10).

Brain MRI is thought to be a more sensitive tool to assess the extent of myelination, as it can detect important changes in 
brain structure in patients with hypothyroidism. For example, neonates with clinically untreated hypothyroidism have been shown to have abnormal brain metabolite changes on magnetic resonance spectroscopy (11) and a reversal of abnormal myelination with thyroxine therapy was documented using proton magnetic resonance spectroscopy in three patients with congenital hypothyroidism (12). Diffusion tensor imaging (DTI) makes it possible to observe WM pathways before myelination is evident on conventional MRI (13), enabling measurements of apparent diffusion coefficients (ADC) and fractional anisotrophy (FA), which are sensitive to the integrity and organization of the WM microstructure. DTI quantification requires computation of a tensor, which is a mathematical description of a three-dimensional ellipsoid depicting the magnitude and orientation of diffusion in individual voxels. The tensor is associated with three corresponding orientational vectors (eigenvectors, $\lambda 1, \lambda 2, \lambda 3$ ), describing the diffusion ellipsoid by its major axes. The eigenvalue average, or trace, reflects the magnitude of diffusion, referred to as mean diffusivity (MD) or the ADC. The extent to which one eigenvalue, $\lambda 1$, dominates the other two, $\lambda 2$ and $\lambda 3$, determines the degree of anisotropy, that is, the degree of orientational preference within a voxel, typically measured as FA, ranges between 0 and 1 on a normalized scale (14). The largest eigenvalue, $\lambda 1$, is the axial (or longitudinal) diffusivity and reflects axonal integrity, whereas $\lambda 2$ and $\lambda 3$ quantify radial (or transverse) diffusivity, $\lambda \mathrm{T}=(\lambda 2+\lambda 3) / 2$, and reflect myelin integrity (15). Thus, disruption of the WM microstructure as detected by DTI can reflect compromised myelin, cytoskeletal structure, and axonal density. Furthermore, MRI with DTI metrics can provide an opportunity to investigate whether measures of brain microstructure reflecting the extent of myelination differ between preterm infants with and without TH dysfunction. Therefore, we aimed to investigate how alterations in brain microstructure affect neurodevelopment after 2 years of follow-up.

Several methods are used determine whether an infant is developing normally, including neurological examinations, parent questionnaires, developmental screening techniques such as the Denver Developmental Screening test II, the CAT/CLAMS, and neurodevelopmental assessments such as the Bayley Scales of Infant Development, third edition (BSID-III), the Gesell Developmental schedules, and the Mullen Scales of Early Learning. Among these assessment tools, the BSID-III require only $45 \mathrm{~min}$ to an hour to complete, however they require an advanced level of training and expertise to administer. In clinics that follow the growth and neurodevelopment of low birth weight infants, the infants may receive either a neurological examination or a developmental assessment.

In this study, we aimed to investigate the impacts of $\mathrm{TH}$ in early [gestational age (GA), <30 weeks] and late (GA, $\geq 30$ weeks) preterm infants, and examine correlations among serum TH level, neurodevelopmental outcomes and MRI with DTI metrics. Therefore, there were three major aims: i) To assess the correlation of thyroid stimulating hormone (TSH) levels at different time points after birth, and neurodevelopmental outcomes at 2 year follow-up; ii) to further investigate whether thyroid status was associated with DTI scalars related to brain microstructure at term equivalence; and iii) to determine whether DTI metrics are a sensitive marker for adverse neurodevelopmental outcomes at 2 years of age.

\section{Patients and methods}

Patient collection. In this prospective cohort study, we enrolled preterm infants with a GA of 23-36 weeks who were admitted to our neonatal intensive care unit (NICU) without perinatal stressful events or asphyxia based on previous study (16). The exclusion criteria were: i) Intrapartum distress including placental abruption, thick meconium amniotic fluid and/or abnormal fetal heart beat (e.g., bradycardia, $<100$ beats/min); ii) Apgar score at the 5th min, $\leq 7$; iii) umbilical cord blood $\mathrm{pH}, \leq 7.15$; iv) umbilical artery base excess, $>12$; v) those requiring bag and mask ventilation for at least 1 min immediately after birth; vi) multi-organ involvement (brain, heart, kidney); vii) those requiring resuscitation within $1 \mathrm{~h}$ of birth; viii) abnormal neurological examination results during NICU stay; ix) abnormal electroencephalography, abnormal head ultrasound, or abnormal neuroimaging findings during NICU stay; $\mathrm{x}$ ) those who presented with any congenital malformation or abnormal genetic conditions (e.g., trisomy 18, trisomy 21 , fragile $\mathrm{X}$ syndrome); and $\mathrm{xi}$ ) those with inborn errors of metabolism (e.g., phenylketonuria, or maple syrup urine disease). The infants of mothers who had thyroid disease, used thyroxin or anti-thyroid drugs during pregnancy were also excluded. The Ethics Committee of Kaohsiung Chang Gung Memorial Hospital (Kaohsiung, Taiwan) approved the study, and written informed consent was obtained from the parents or guardians of the newborns (IRB no. 100-2025A3).

The study subjects were divided into two groups according to GA: The early preterm group ( $<30$ weeks) and late preterm group ( $\geq 30$ weeks). Clinical data, including sex, GA, Apgar score at 1 and $5 \mathrm{~min}$, arterial $\mathrm{pH}$, delivery mode and birth body weight were collected.

Thyroid function tests in the preterm infants. A blood sample was collected from each infant in both groups for gas analysis immediately after admission to the NICU to determine the level of TSH using a heel stick blood test. The level of TSH was determined using drops of blood collected on filter paper on the initial day of admission (range, $1-2 \mathrm{~h}$ ), and at $18 \mathrm{~h}$ (range, 1-2 h) and $24 \mathrm{~h}$ of admission (range, 2-4 h) which were stored at $-70^{\circ} \mathrm{C}$ in sealed bags with desiccant until being processed. All procedures were performed by the same biochemist who was blinded to the patients' data. The level of TSH was determined from the dried blood spots at a university hospital newborn screening program and expressed in $\mu \mathrm{U} / \mathrm{ml}$. The cut-off value of TSH used to define hypothyroxinemia in the blood spot tests was $9 \mu \mathrm{U} / \mathrm{ml}$ based on reference range of Taiwan Newborn Screening Center. The blood spot analyses were done using Immuchem ${ }^{\text {TM }}$ Neonatal TSH-MW ELISA (bulk kit-20 plates) and RIA kits (500 tubes kit) (MP Biochemicals, Santa Ana, CA, USA). If the results were abnormal, the level was rechecked after 2 weeks. The patients with a normal level in the second exam were defined as having 'transient' hypothyroidism, and those with abnormal results in the second exam were defined as having 'persistent' hypothyroidism requiring thyroxine replacement therapy.

MRI study protocol. The study group and control group underwent conventional MRI with DTI at a postmenstrual term-equivalent age. Parental informed consent was always 
obtained before the examination. The data were evaluated retrospectively by an experienced neuro-radiologist.

Images were acquired using standard scanning protocols on a 1.5-T GE Echo Speed scanner (GE Medical Systems, Milwaukee, WI, USA) including T1-W (spin echo, TR/TE 500/11 msec) and T2-W sequences (spin echo, TR/TE 3,000/120 msec) with a slice thickness of $4 \mathrm{~mm}$ and 0.4-mm gap. For DTI, an echo planar sequence with diffusion gradients $\left(b=1,000 \mathrm{sec} / \mathrm{mm}^{2}\right)$ applied in 25 non-collinear directions was used with a slice thickness of $3 \mathrm{~mm}$ and no gap. An average of 20 slices was recorded within 4 min using TR/TE 9,150/98-91 msec. The field of view was $20 \mathrm{~cm}$, the scan matrix was $128 \times 128$, and the reconstruction matrix was $256 \times 256$. All of the infants were scanned using a magnetic resonance-compatible incubator with a specialized high-sensitivity neonatal head coil (LMT Medical Systems GmbH, Lübeck, Germany) that allowed for DTI imaging at high spatial resolution and high signal-to-noise ratio. The incubator provided controlled temperature and humidity, and the infants were sedated with chloral hydrate $50 \mathrm{mg} / \mathrm{kg} 30 \mathrm{~min}$ before the examination and their position was fixed using a vacuum pillow. This set-up allowed for imaging in this vulnerable patient population in a stable and safe microenvironment. We used moldable earplugs and neonatal earmuffs to reduce the noise, and pads around the infant's head kept movement to a minimum. The DTI scalars we chose were listed as followings: Radial diffusivity (RD), MD, axial diffusivity (AD), and FA are DTI scalars which are sensitive markers for measuring the biological microstructure of WM. AD tends to be variable in WM pathology and tends to increase with brain maturation. The tracts selected for quantization in the study included commissural tracts [corpus callosum (CC): Splenium and genu], projection tracts including those of the posterior limb of the internal capsule (PLIC), the anterior limb of the internal capsule (ALIC), and association tracts [external capsule (EC)]. Regions of interest (ROIs) of the DTI measurements were identified from multiple ROIs positioned bilaterally within individual WM tracts. For placement, we used standard-sized, round-shaped 16-pixel ROIs.

Neurodevelopmental evaluation. At 6, 12, 18, and 24 months corrected age, the BSID-III was administered by experienced testers (17). The BSID-III contains two main scales: The mental developmental index (MDI) and psychomotor developmental index (PDI). MDI and PDI scores of $100 \pm 15$ represent the mean \pm 1 SD. BSID-PDI had designed to assess cognition, and the BSID-MDI had designed to evaluate motor outcome at 2-year follow-up. To assess whether DTI scores were correlated to motor outcomes, we defined the infants receiving physical and occupational therapy as the 'Neuromotor dysfunction' group, and the other healthy infants as the 'without neuromotor dysfunction' group.

Statistical analysis. Statistical analysis was performed using SPSS software version 18.0 (SPSS, Inc., Chicago, IL, USA). Differences in categorical variables between two groups were analyzed using the independent Student's t-test. TSH levels at different time points were analyzed using one-way ANOVA with repeat measurements. The correlations between neurodevelopment, MD and GAs were analyzed by Pearson's
Table I. Clinical information of enrolled patients $(n=81)$.

\begin{tabular}{lccc}
\hline Variables & $\begin{array}{c}\text { 30 weeks } \\
(\mathrm{n}=17)\end{array}$ & $\begin{array}{c}>30 \text { weeks } \\
(\mathrm{n}=64)\end{array}$ & P-value \\
\hline Sex (n) & & & \\
Male & 9 & 30 & 0.786 \\
Female & 8 & 34 & \\
Apgare score & & & \\
1 min $<6$ (n) & 6 & 9 & \\
5 min $<6$ (n) & 0 & 2 & $0.005^{\text {a }}$ \\
Delivery mode & & 53 & \\
CS & 8 & 11 & $0.021^{\text {a }}$ \\
NSD & 9 & $1,287.3 \pm 388.1$ & \\
Birth weight $(\mathrm{g})$ & $1,032.5 \pm 32.5$ & & \\
Arterial pH & & 13 & \\
pH $<7.25$ & 3 & &
\end{tabular}

${ }^{\mathrm{a}} \mathrm{P}<0.05 ; \mathrm{CS}$, caesarean section; NSD, normal spontaneously delivery.

correlation analysis. The correlation among plasma TSH levels, DTI metrics of ROIs and neurodevelopmental outcomes were analyzed using bivariate correlation analysis. A P-value $<0.05$ was considered to indicate statistical significance.

\section{Results}

A total of 81 premature infants were enrolled in this study with a GA ranging from 23-36 weeks. Infants in the late preterm group were significantly heavier than the early preterm group $(1,287.3 \pm 388.1$ vs. $1,032.5 \pm 32.5 \mathrm{~g} ; \mathrm{P}<0.001)$ and were more likely to be delivered by cesarean section (CS) (Table I). Caues of CS were summarized in Table II. We have only 8 patients with GA $<30$ weeks receiving $\mathrm{CS}$, and most of the causes were contributed to maternal health problems. In the contrast, we have 54 patients with GA > 30 weeks receiving CS, and the causes were contributed eventfully to fetal and maternal problems (Table II). There were no significant differences in sex, Apgar score at 1 and $5 \mathrm{~min}$, and initial umbilical arterial $\mathrm{pH}$ between the two groups.

There were also no significant differences in mean TSH values between the groups at 0,18 , or $24 \mathrm{~h}$ of admission. The mean MDI scores were similar between the two groups from 6 months to 24 months of follow-up, and the mean PDI scores were comparable between the two groups until 18 months corrected age. The early preterm group had significantly lower PDI scores than the late preterm group at 24 months of follow-up (93 \pm 31 vs. 107 \pm 31$)$. The rates of HTT were $4.5,0$, and $4.5 \%$ at TSH0, TSH18, and TSH 24 in the early preterm group, and $20.3,8.5$, and $3.4 \%$ in the late preterm group, respectively. All of the infants with abnormal results had normal results in the second examination, which indicated all of the preterm with HTT in our study had transient hypothyroidism (Table III). Multivariate analysis had been performed to analyze the factors related to neurodevelopmental outcome. The data demonstrated that GA is correlated to motor function (PDI) at 2 year follow-up (Fig. 1). However, neither GA 
Table II. Causes of cesarean section in study subjects.

\begin{tabular}{llc}
\hline $\begin{array}{l}\text { Causes of cesarean } \\
\text { delivery }\end{array}$ & $\begin{array}{c}\text { GA } \leq 30 \text { weeks } \\
(\mathrm{CS}, \mathrm{n}=8)(\mathrm{n}, \%)\end{array}$ & $\begin{array}{c}\text { GA }>30 \text { weeks } \\
(\mathrm{CS}, \mathrm{n}=54)(\mathrm{n}, \%)\end{array}$ \\
\hline $\begin{array}{l}\text { Fetal problems (total) } \\
\text { Fetal distress }\end{array}$ & $3(37.5)$ & $26(48.1)$ \\
Premature delivery & $0(12.5)$ & $9(16.7)$ \\
Fetal malposition & $0(0)$ & $9(16.7)$ \\
Cord prolapse & $0(0)$ & $3(5.5)$ \\
Twin-twin transfusion & $1(12.5)$ & $1(1.8)$ \\
syndrome & & $0(0)$ \\
Oligohydramnios & $0(0)$ & $1(1.9)$ \\
Chorioamnionitis & $0(0)$ & $1(1.9)$ \\
Fetal growth arrest & $0(0)$ & $2(3.7)$ \\
Maternal problems (total) & $5(62.5)$ & $28(51.9)$ \\
Multiple pregnancies & $0(0)$ & $10(18.5)$ \\
Placenta problems & $1(12.5)$ & $6(11.1)$ \\
Preeclampsia & $4(50)$ & $5(13.0)$ \\
Maternal medical & $0(0)$ & \\
crisis & & \\
\hline
\end{tabular}

GA, gestational age; CS, cesarean section.

nor DTI indices is correlated to cognition function (data not shown).

To investigate the association between $\mathrm{TH}$ function and neurodevelopmental outcomes in later life, we analyzed correlations between TSH levels at 0,18 , and $24 \mathrm{~h}$ of admission (TSH0, TSH18, TSH24) and MDI/PDI scores using bivariate correlation analysis. There were no significant correlations between TSH levels and developmental indices. Some r-values for TSH time point are positive and some are negative in Table IV. This was possibly due to the randomness of result but not biological relationship since the r-value was small and the distribution of positive or negative data was not related to chronologic age. BSID-III MDI scoreswere positively correlated with GA at 2 years of follow-up. The late preterm group had higher MDI scores compared to the early preterm group. Furthermore, BSID-III PDI scoreswere positively correlated with GA throughout the 2 years of follow-up. The late preterm group had higher PDI scores compared to the early preterm group, suggesting that GA but not thyroid function determined the long-term neurological outcomes in the premature infants (Table IV).

Nineteen cases withdrew from MRI study because of difficulty in sedating with oral chloral hydrate. Ten poor-quality records were discarded because of motion artifact. Twenty four cases terminated participation for fear of side effect of chloral hydrate. Another twenty cases lost of follow-up from prematurity follow-up program. Only 8 reliable DTI indices data could be available. Our data showed that AD $(\lambda 1)$ was significantly decreased in the splenium of the CC in the early preterm group, and that FA was also significantly decreased in the early preterm group in the anterior and posterior limbs of the internal capsule and the EC. In our observation, FA and AD decrease in these ROIs did not correlate to adverse neuromotor
Table III. Illustrations of TSH values, mental index, and performance index in enrolled patients $(n=81)$.

\begin{tabular}{|c|c|c|c|}
\hline Variables & $\begin{array}{c}\leq 30 \text { weeks } \\
\quad(n=17)\end{array}$ & $\begin{array}{l}>30 \text { weeks } \\
\quad(n=64)\end{array}$ & P-value \\
\hline \multicolumn{4}{|l|}{$\mathrm{TSH}$} \\
\hline TSHO & $7.3 \pm 0.22$ & $9.0 \pm 0.1$ & 0.283 \\
\hline TSH18 & $3.6 \pm 0.09$ & $3.9 \pm 0.04$ & 0.688 \\
\hline TSH24 & $3.3 \pm 0.14$ & $3.5 \pm 0.04$ & 0.762 \\
\hline \multicolumn{4}{|c|}{$\begin{array}{l}\text { Hyperthyrotropinemia } \\
(\mathrm{TSH}>9 \mu \mathrm{U} / \mathrm{ml})\end{array}$} \\
\hline $0 \mathrm{~h}[\mathrm{n}(\%)]$ & $1(4.5)$ & $12(20.3)$ & \\
\hline $18 \mathrm{~h}[\mathrm{n}(\%)]$ & $0(0)$ & $5(8.5)$ & \\
\hline $24 \mathrm{~h}[\mathrm{n}(\%)]$ & $1(4.5)$ & $2(3.4)$ & \\
\hline \multicolumn{4}{|l|}{ MDI (months) } \\
\hline 6 & $105 \pm 33$ & $107 \pm 34$ & 0.328 \\
\hline 12 & $95 \pm 29$ & $104 \pm 22$ & 0.448 \\
\hline 18 & $104 \pm 33$ & $103 \pm 24$ & 0.098 \\
\hline 24 & $92 \pm 31$ & $101 \pm 30$ & 0.071 \\
\hline \multicolumn{4}{|l|}{ PDI (months) } \\
\hline 6 & $85 \pm 27$ & $97 \pm 31$ & 0.496 \\
\hline 12 & $91 \pm 27$ & $97 \pm 21$ & 0.851 \\
\hline 18 & $97 \pm 31$ & $102 \pm 24$ & 0.105 \\
\hline 24 & $93 \pm 31$ & $107 \pm 31$ & $0.049^{\mathrm{a}}$ \\
\hline
\end{tabular}

${ }^{\mathrm{a}} \mathrm{P}<0.05$. TSH, thyroid stimulating hormone; TSH0, TSH level at initially admitted to neonatal intensive care unit (NICU); TSH18, TSH level at $18 \mathrm{~h}$ of admission to NICU; TSH24, TSH level at $24 \mathrm{~h}$ of admission to NICU; MDI, mental developmental index; PDI, psychomotor developmental index.

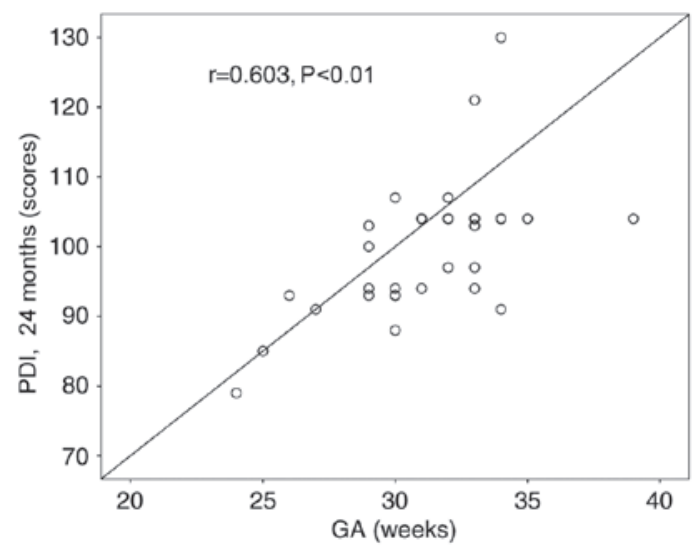

Figure 1. GA significantly impact on motor function at 2 year follow-up by multivariate analysis. GA, gestational age; PDI, psychomotor developmental index.

in our study (Table V). As for the correlation between TSH and DTI scalars, our data demonstrated no significant correlation between TSH and DTI scalars except the AD $(\lambda 1)$ value over the splenium of the $\mathrm{CC}$, which showed negatively correlated with TSH0 (Fig. 2A), while the FA value over the anterior limb of the internal capsule was positively correlated with TSH0 (Fig. 2B). However, these changes in DTI scalars in ROIs did 
Table IV. Correlation between TSH levels, GA and developmental index by bivariate correlation analysis.

\begin{tabular}{lrrrr}
\hline $\begin{array}{l}\text { Developmental } \\
\text { index (postnatal } \\
\text { age) }\end{array}$ & \multicolumn{4}{c}{ Bivariate correlation analysis $\mathrm{r}$} \\
\cline { 2 - 5 } & TSH0 & TSH18 & TSH24 & GA \\
\hline MDI (months) & & & & \\
6 & -0.138 & -0.071 & -0.042 & 0.000 \\
12 & 0.035 & 0.066 & -0.029 & $0.340^{\mathrm{a}}$ \\
18 & 0.008 & -0.009 & 0.080 & 0.150 \\
24 & 0.114 & 0.158 & 0.132 & $0.626^{\mathrm{b}}$ \\
PDI (months) & & & & \\
6 & 0.156 & -0.164 & -0.007 & $0.238^{\mathrm{c}}$ \\
12 & 0.086 & 0.012 & 0.028 & $0.414^{\mathrm{b}}$ \\
18 & -0.013 & -0.054 & -0.083 & $0.431^{\mathrm{b}}$ \\
24 & -0.025 & 0.099 & 0.022 & $0.608^{\mathrm{b}}$ \\
\hline
\end{tabular}

${ }^{\mathrm{a}} \mathrm{P}<0.01 ;{ }^{\mathrm{b}} \mathrm{P}<0.001 ;{ }^{\mathrm{c}} \mathrm{P}<0.05$. TSH, thyroid stimulating hormone; GA, gestational age; TSHO, TSH level at initially admitted to neonatal intensive care unit (NICU); TSH18, TSH level at $18 \mathrm{~h}$ of admission to NICU; TSH24, TSH level at $24 \mathrm{~h}$ of admission to NICU MDI, mental developmental index; PDI, psychomotor developmental index.

not show clinical significance in predicting the long-term neuromotor outcomes. MD is an inverse measure of the membrane density, and is sensitive to cytoedema and cellular necrosis. We found that MD in the ROIs of the genus and splenium of the $\mathrm{CC}$ were significantly higher in the infants with motor dysfunction group. In addition, MD in the ROIs of the right side ALIC was relatively higher in the motor dysfunction group, although without statistical significance (Table VI). The illustrative fig. was demonstrated in Fig. 3.

\section{Discussion}

In this study, we recorded TSH levels of infants in early and late preterm groups and analyzed the association between TSH levels and neurodevelopmental outcomes according to the BSID-III. All of our patients with HTT had transient changes not requiring thyroxine therapy, and none of the infants had detrimental neurodevelopmental outcomes at 2 years of age, and GA but not TSH level determined neuromotor outcomes in the infants at 2 years of age. We also analyzed the association between regional WM microstructural development and TSH level, and found that FA increased with TSHO levels over the ALIC, while AD $(\lambda 1)$ decreased with TSH0 levels over the SC. We further found that FA values increased at the genus and splenium of the $\mathrm{CC}$, and that MD value tended to decrease with increasing age, which is consistent with a previous report (18). We also found lower MD scores in the patients with motor dysfunction.

$\mathrm{TH}$ are known to play a critical role in the growth, development, and maturation of the nervous system. Animal experiments have suggested that $\mathrm{TH}$ are also necessary for myelination, a process essential for motor function. Congenital hypothyroidism in newborns has been reported to have an adverse impact on intelligent quotient even after thyroxine
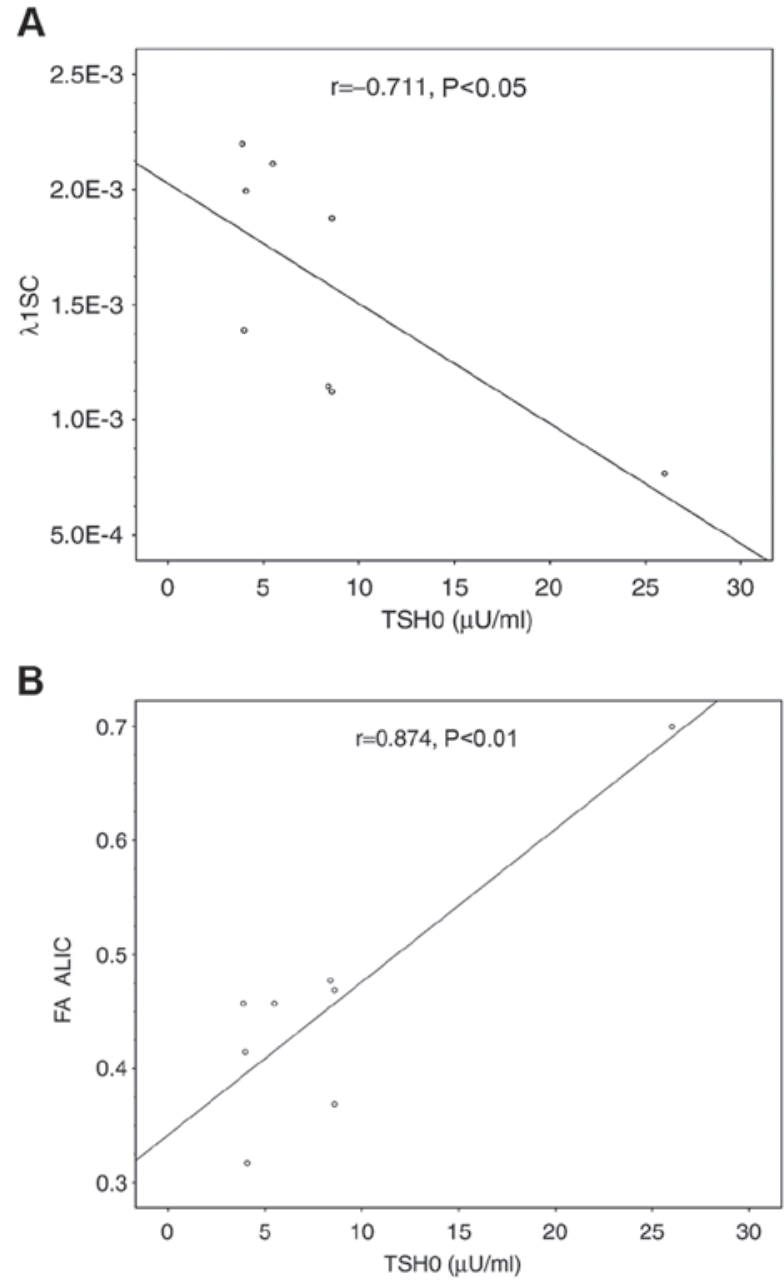

Figure 2. Correlations between DTI indices and TSH0. TSH0 was found adversely correlated to $\mathrm{AD}(\lambda 1)$ in the splenium of the corpus callosum (A) and positively correlated to FA in the ALIC (B). DTI, diffusion tensor imaging; TSH0, TSH level at initially admitted to neonatal intensive care unit; AD, axial diffusivity; FA, fractional anisonotrophy; ALIC, bilateral sides of anterior limb of internal capsule; SC, splenium of corpus callosum.

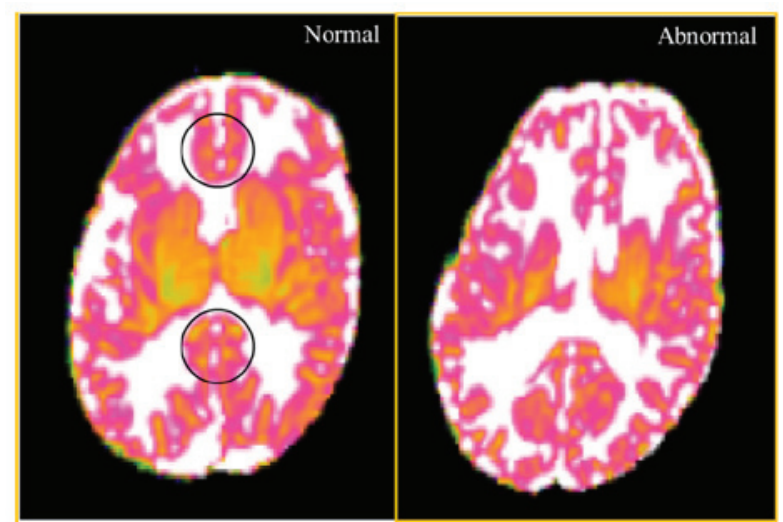

Figure 3. Illustrative feature demonstrated the abnormal MD value (in red) in patients with neuromotor dysfunction. The left column represented preterm infants lived without neuromotor dysfunction. The upper circle indicated genus of corpus callosum, while the lower circle indicated splenium of corpus callosum. MD, mean diffusivity.

replacement therapy. Thus, even transiently low levels of thyroxine can be a risk factor for adverse neurodevelopmental 
Table V. Comparison of diffusion scalars between premature babies $(n=8)$ with gestational age $<30$ weeks and $\leq 30$ weeks.

\begin{tabular}{|c|c|c|c|}
\hline Variables & $\leq 30$ weeks $(n=3)$ & $>30$ weeks $(\mathrm{n}=5)$ & P-value \\
\hline \multicolumn{4}{|l|}{ Radial diffusivity } \\
\hline Genum of $\mathrm{CC}$ & $0.001158 \pm 0.000669$ & $0.001313 \pm 0.000667$ & 0.279 \\
\hline Splenium of CC & $0.001173 \pm 0.000677$ & $0.001439 \pm 0.00072$ & 0.212 \\
\hline ALIC & $0.002044 \pm 0.00118$ & $0.002234 \pm 0.00117$ & 0.619 \\
\hline PLIC & $0.001713 \pm 0.000989$ & $0.001729 \pm 0.00865$ & 0.929 \\
\hline $\mathrm{EC}$ & $0.001944 \pm 0.001123$ & $0.002451 \pm 0.001225$ & 0.255 \\
\hline \multicolumn{4}{|l|}{ Mean diffusivity } \\
\hline Genum of CC & $0.001538 \pm 0.000769$ & $0.001233 \pm 0.000712$ & 0.106 \\
\hline Splenium of CC & $0.001634 \pm 0.000817$ & $0.001433 \pm 0.000827$ & 0.297 \\
\hline ALIC & $0.002701 \pm 0.00135$ & $0.002389 \pm 0.001379$ & 0.394 \\
\hline PLIC & $0.002476 \pm 0.001238$ & $0.001685 \pm 0.000973$ & 0.369 \\
\hline $\mathrm{EC}$ & $0.002787 \pm 0.001393$ & $0.002387 \pm 0.001378$ & 0.297 \\
\hline \multicolumn{4}{|c|}{ Axial diffusivity $(\lambda 1)$} \\
\hline Genum of CC & $0.001594 \pm 0.00092$ & $0.001871 \pm 0.000936$ & 0.076 \\
\hline Splenium of CC & $0.001638 \pm 0.000946$ & $0.002163 \pm 0.001082$ & $0.027^{\mathrm{a}}$ \\
\hline ALIC & $0.002694 \pm 0.001555$ & $0.00311 \pm 0.001562$ & 0.160 \\
\hline PLIC & $0.002828 \pm 0.001633$ & $0.003124 \pm 0.001562$ & 0.160 \\
\hline $\mathrm{EC}$ & $0.002591 \pm 0.001496$ & $0.003274 \pm 0.001637$ & 0.087 \\
\hline \multicolumn{4}{|c|}{ Fractional anisotrophy } \\
\hline Genum of CC & $0.151736 \pm 0.087605$ & $0.227086 \pm 0.113543$ & 0.078 \\
\hline Splenium of CC & $0.177009 \pm 0.102196$ & $0.2450878 \pm 0.1225439$ & 0.105 \\
\hline ALIC & $0.264688 \pm 0.152818$ & $0.3678843 \pm 0.1839421$ & $0.045^{\mathrm{a}}$ \\
\hline PLIC & $0.339957 \pm 0.196274$ & $0.551738 \pm 0.275869$ & $0.043^{\mathrm{a}}$ \\
\hline $\mathrm{EC}$ & $0.288964 \pm 0.166833$ & $0.3782525 \pm 0.1891263$ & $0.041^{\mathrm{a}}$ \\
\hline
\end{tabular}

${ }^{\mathrm{a}} \mathrm{P}<0.05$. CC, corpus callosum; ALIC, anterior limb of internal capsule; PLIC, posterior limb of internal capsule; EC, external capsule

Table VI. Correlation between DTI indices and neuromotor function.

\begin{tabular}{|c|c|c|c|}
\hline & Without neuromotor dysfunction $(n=5)$ & With neuromotor dysfunction $(\mathrm{n}=3)$ & P-value \\
\hline MD GC & $0.0014(0.0012-0.0015)$ & $0.0016(0.00156-0.00162)$ & $0.024^{\mathrm{a}}$ \\
\hline MD SC & $0.0016(0.0013-0.0016)$ & $0.0017(0.0017-0.00171$ & $0.024^{\mathrm{a}}$ \\
\hline MD ALICR & $0.0011(0.00100-0.00135)$ & $0.00138(0.00133-0.00139)$ & 0.051 \\
\hline $\mathrm{AD}(\lambda 1) \mathrm{SC}$ & $0.00148(0.0010-0.0015)$ & $0.0011(0.0007-0.0015)$ & 0.456 \\
\hline FA ALIC & $0.4573(0.3688-0.4591)$ & $0.4573(0.3660-0.4589)$ & 0.546 \\
\hline FA GC & $0.2656(0.1919-0.3544)$ & $0.1825(0.1825-0.1936)$ & 0.294 \\
\hline FA SC & $0.2878(0.2076-0.3797)$ & $0.1975(0.1975-0.2037)$ & 0.177 \\
\hline
\end{tabular}

${ }^{\mathrm{a}} \mathrm{P}<0.05$. The numbers in the parentheses represent the range with an average. MD, mean diffusivity; GC, genus of corpus callosum; SC, splenium of corpus callosum; ALICR, right side anterior limb of internal capsule; AD, axial diffusivity; FA, fractional anisonotrophy; ALIC, bilateral sides of anterior limb of internal capsule.

outcomes in preterm infants $(19,20)$. Three screening strategies are used for congenital hypothyroidism: A primary TSH method, a primary thyroxine method, and a combined primary TSH and thyroxine method. The newborn screening test in Taiwan uses a primary TSH method instead of free thyroxine level. The cut-off value of TSH is defined as $9 \mu \mathrm{U} / \mathrm{ml}$, which has been reported to be sensitive for detecting newborns with congenital hypothyroidism (21). Transient HTT with an elevated TSH level is common in preterm infants, and can lead to transient or permanent hypothyroidism. Although our study provided only TSH value without free thyroxin level for sake of facility limitation, all of our cases encountered transient HTT were assumed to have only transient hypothyroidism since none of our enrolled cases had permanent hypothyroidism 
requiring thyroxine supplements. The fertility rate in Taiwan is 0.9 which is one of the lowest globally, and that is why only 81 cases were enrolled in this study.

Dammann and Leviton proposed that both, a two-component model, play an important role in WM damage in preterm infants (22). The insult model suggests that hypoxic-ischemic and/or infection causes elevated inflammatory responses, which consequently results in WM injury. The developmentally-regulated endogenous protection theory includes three components of vulnerability associated with immaturity: vascular/ependymal factors, oligodendroglial development factors, and the lack of certain developmentally-regulated endogenous protectors such as neurotrophins, oligotrophins (such as nerve growth factor, neurotrophin-3, and ciliary-neurotrophic factor), hormones (of which $\mathrm{TH}$ are best known), and cytokines (interleukin-6 family) in preterm infants (22). TH promotes the maturation of oligodendrocytes past a period of vulnerability, and may protect against WM injury due to hypoxic-ischemic insults in developing brains (23). HTT is only a surrogate for thyroid deficiency, however, it has been reported to be linked to inflammation and thyroid deficiency in neonates (24). It is possible that some of our patients still had HTT at $24 \mathrm{~h}$ postnatal age, indicating that they may have had inflammation severe enough to cause brain damage. Another possibility is that HTT is an expression of immaturity/vulnerability. TSH levels tend to be higher in newborns with a lower GA which reverts to normality at re-examination after 2 weeks $(25,26)$. Although one multicenter study reported tranisient HTT is a risk factor of developing persistent HTT in children with neurodevelopment delays (27), our results revealed that HTT in our study reverts to normality without neurodevelopmental consequences. It was GA, but not thyroid function, determined the long-term neurological outcomes in our premature infants.

MRI can detect the extent of myelination in brain structures in hypothyroidism, and DTI metrics make it possible to observe WM pathways before myelination is evident on conventional MRI (11) $\mathrm{Ng}$ et al reported no differences in brain MRI at term equivalence in DTI metrics either in FA or MD in preterm infants who received LT4 supplements compared with those who had not. However, low ADC values were associated with better WM organization among infants with higher plasma levels of thyroxine (28). In contrast to previous studies, we found that $\mathrm{AD}(\lambda 1)$ values over the splenium of the $\mathrm{CC}$ were negatively correlated to TSH0, while FA values over the anterior limb of the internal capsule were positively correlated to TSH0, although neither reached significance. In addition, our data should be interpreted with caution because there was an outlier case with extremely high TSH level in the analysis. If this case was excluded from the analysis, there was no association between TSH level and DTI metrics.

Rogers et al demonstrated a pattern of changes in diffusion measurements during WM development, with increases in FA and decreases in MD with increasing postmenstrual age (18). In this study, the FA slope in the PLIC was steeper than in other WM regions, which likely reflects early myelination. In addition, ALIC, CC and optic radiations have been demonstrated to have steeper FA slopes with increasing GA (18). Consistent with the study by Roger et al, we also found that FA increased with age in a regionally-specific manner over regions of the ALIC, PLIC and EC. The data indicated the late preterm group had more intact WM integrity over the anterior and posterior limbs of the internal capsule and EC in FA than the early preterm group. To the best of our knowledge, this is the first study to report increases in FA with age in the EC. We also showed that MD tends to decrease with increasing GA, although there was no statistical significance. AD measures water diffusion along the axis of the axon and increases during axonal organization as the axons become more coherently arranged along the main axis. It is largely stable during true myelination as the hydrophobic myelin sheathing restricts diffusion in the perpendicular direction. We found significantly increased AD values with increasing GA in the splenium of the CC.

We next investigated whether the changes in DTI metrics in this study were clinically relevant to the neurodevelopmental outcomes. Lower FA and higher MD values in the genu of the $\mathrm{CC}$ have been reported to be correlated with slower gait velocity, and lesion studies have indicated that the genus WM mediates motor coordination (29,30). Our findings are partially consistent with the previous results. The patients with motor dysfunction who needed rehabilitation in our study had significantly higher MD values at the ROIs of the genu and splenium of the CC than those who did not need rehabilitation. Of note, relatively higher MD values were obtained at the right side of the ALIC in the patients with motor dysfunction, although the results did not reach statistical significance. In addition, Rose et al reported that near-term ALIC MD values were correlated with BSID-III fine motor scores (29). We did not observe increases in FA in the genu and splenium of the $\mathrm{CC}$ in the patients with motor dysfunction, which may be due to the limited number of patients. There are four major limitations to this study. First, we lacked information about thyroxine levels at the time TSH levels were determined, and thus we could not differentiate HTT due to thyroxine deficiency or host medical conditions. Second, the children had to survive until 2 years of age and return for a developmental assessment to be included in this study, and the patients who died with preterm comorbidities were not included. As these children differed from the survivors, the requirements for survival may have introduced bias. Third, only eight patients participated in the MRI study at a postmenstrual age of 40 weeks due to concerns of guardians with regards the risk of sedation for the MRI studies. In addition, a Dixon's Q test was run and identified the outlier data in Fig. $2(\mathrm{P}=0.002)$. We still kept the outlier data in Fig. 2 since babies possessing TSH with an outlier value at initial of admission needed to be double checked at term-equivalent age. This is current newborn screening policy in Taiwan. We need more study subjects to reach a significant statistical power. Fourth, we should be cautious to interpret the association among TSH0, $\mathrm{AD}$ and FA since removing the outlier from the analyses leads to non-significant findings. In conclusion, GA determined the neurodevelopmental outcomes of the premature infants in this study. Transient hypothyroidism is a risk factor for neurodevelopmental outcome, however, it did not play a critical role in neuromotor dysfunction in preterm babies. Our novel findings demonstrated that the infants with neuromotor dysfunction had significantly higher MD values at the ROIs in the genu and splenium of the CC. A further investigation with a larger sample size is necessary to validate our results. 


\section{Acknowledgements}

This study was supported by the Research Support Scheme of Chang Gung Memorial Hospital (grant no. CMRPG8B0721). We thank the Biostatistics Center of Kaohsiung Chang Gung Memorial Hospital for assistance with the statistics. The funding source played no role in the study design, in the collection, analysis and interpretation of data; in the writing of the manuscript; and in the decision to submit the manuscript for publication.

\section{References}

1. Patel J, Landers K, Li H, Mortimer RH and Richard K: Thyroid hormones and fetal neurological development. J Endocrinol 209: $1-8,2011$

2. MeijerWJ, Verloove-Vanhorick SP, Brand R and van den Brande JL: Transient hypothyroxinaemia associated with developmental delay in very preterm infants. Arch Dis Child 67: 944-947, 1992.

3. Ahmed OM, El-Gareib AW, El-Bakry AM, Abd El-Tawab SM and Ahmed RG: Thyroid hormones states and brain development interactions. Int J Dev Neurosci 26: 147-209, 2008.

4. Ahmed RG: Hypothyroidism and brain developmental players. Thyroid Res 8: 2, 2015.

5. Den Ouden AL, Kok JH, Verkerk PH, Brand R and VerlooveVanhorick SP: The relation between neonatal thyroxine levels and neurodevelopmental outcome at age 5 and 9 years in a national cohort of very preterm and/or very low birth weight infants. Pediatr Res 39: 142-145, 1996.

6. Vigone MC, Caiulo S, Di Frenna M, Ghirardello S, Corbetta C, Mosca F and Weber G: Evolution of thyroid function in preterm infants detected by screening for congenital hypothyroidism. J Pediatr 164: 1296-1302, 2014.

7. van Wassenaer AG and Kok JH: Hypothyroxinaemia and thyroid function after preterm birth. Semin Neonatol 9: 3-11, 2004.

8. Schoonover CM, Seibel MM, Jolson DM, Stack MJ, Rahman RJ, Jones SA, Mariash CN and Anderson GW: Thyroid hormone regulates oligodendrocyte accumulation in developing rat brain white matter tracts. Endocrinology 145: 5013-5020, 2004.

9. Sugisaki T, Noguchi T and Tsukada Y: Cerebral myelinogenesis in the Snell dwarf mouse: Stimulatory effects of GH and T4 restricted to the first 20 days of postnatal life. Neurochem Res 10: 767-778, 1985.

10. Korzeniewski SJ, Birbeck G, DeLano MC, Potchen MJ and Paneth N: A systematic review of neuroimaging for cerebral palsy. J Child Neurol 23: 216-227, 2008.

11. Gupta RK, Bhatia V, Poptani H and Gujral RB: Brain metabolite changes on in vivo proton magnetic resonance spectroscopy in children with congenital hypothyroidism. J Pediatr 126: 389-392, 1995.

12. Jagannathan NR, Tandon N, Raghunathan P and Kochupillai N: Reversal of abnormalities of myelination by thyroxine therapy in congenital hypothyroidism: Localized in vivo proton magnetic resonance spectroscopy (MRS) study. Brain Res Dev Brain Res 109: 179-186, 1998.

13. Berman JI, Mukherjee P, Partridge SC, Miller SP, Ferriero DM, Barkovich AJ, Vigneron DB and Henry RG: Quantitative diffusion tensor MRI fiber tractography of sensorimotor white matter development in premature infants. Neuroimage 27: 862-871, 2005.

14. Basser PJ and Pierpaoli C: Microstructural and physiological features of tissues elucidated by quantitative-diffusion-tensor MRI. J Magn Reson B 111: 209-219, 1996.
15. Song SK, Sun SW, Ramsbottom MJ, Chang C, Russell J and Cross AH: Dysmyelination revealed through MRI as increased radial (but unchanged axial) diffusion of water. Neuroimage 17: 1429-1436, 2002.

16. Pereira DN and Procianoy RS: Effect of perinatal asphyxia on thyroid-stimulating hormone and thyroid hormone levels. Acta Paediatr 92: 339-345, 2003.

17. D'Agostino JA: An evidentiary review regarding the use of chronological and adjusted age in the assessment of preterm infants. J Spec Pediatr Nurs 15: 26-32, 2010.

18. Rogers CE, Smyser T, Smyser CD, Shimony J, Inder TE and Neil JJ: Regional white matter development in very preterm infants: Perinatal predictors and early developmental outcomes. Pediatr Res 79: 87-95, 2016.

19. Simic N, Asztalos EV and Rovet J: Impact of neonatal thyroid hormone insufficiency and medical morbidity on infant neurodevelopment and attention following preterm birth. Thyroid 19: 395-401, 2009.

20. Reuss ML, Paneth N, Pinto-Martin JA, Lorenz JM and Susser M: The relation of transient hypothyroxinemia in preterm infants to neurologic development at two years of age. N Engl J Med 334: 821-827, 1996.

21. Olivieri A, Fazzini C and Medda E; Italian Study Group for Congenital Hypothyroidism: Multiple factors influencing the incidence of congenital hypothyroidism detected by neonatal screening. Horm Res Paediatr 83: 86-93, 2015.

22. Dammann O and Leviton A: Brain damage in preterm newborns: Might enhancement of developmentally regulated endogenous protection open a door for prevention? Pediatrics 104: 541-550, 1999.

23. Hung PL, Huang CC, Huang HM, Tu DG and Chang YC: Thyroxin treatment protects against white matter injury in the immature brain via brain-derived neurotrophic factor. Stroke 44 . 2275-2283, 2013.

24. Soto-Rivera CL, Fichorova RN, Allred EN, Van Marter LJ, Shah B, Martin CR, Agus MS and Leviton A: The relationship between TSH and systemic inflammation in extremely preterm newborns. Endocrine 48: 595-602, 2015.

25. Kaye C; Committee on Genetics, Accurso F, La Franchi S, Lane PA, Northrup H, Pang S and Schaefer GB: Introduction to the newborn screening fact sheets. Pediatrics 118: 1304-1312, 2006.

26. Gaudino R, Garel C, Czernichow P and Léger J: Proportion of various types of thyroid disorders among newborns with congenital hypothyroidism and normally located gland: A regional cohort study. Clin Endocrinol (Oxf) 62: 444-448, 2005.

27. Cuestas E, Gaido MI and Capra RH: Transient neonatal hyperthyrotropinemia is a risk factor for developing persistent hyperthyrotropinemia in childhood with repercussion on developmental status. Eur J Endocrinol 172: 483-490, 2015

28. Ng SM, Turner MA, Gamble C, Didi M, Victor S, Atkinson J, Sluming V,Parkes LM, Tietze A, Abernethy LJ and Weindling AM: Effect of thyroxine on brain microstructure in extremely premature babies: Magnetic resonance imaging findings in the TIPIT study. Pediatr Radiol 44: 987-996, 2014.

29. Rose J, Cahill-Rowley K, Vassar R, Yeom KW5, Stecher X, Stevenson DK, Hintz SR and Barnea-Goraly N: Neonatal brain microstructure correlates of neurodevelopment and gait in preterm children 18-22 mo of age: An MRI and DTI study. Pediatr Res 78: 700-708, 2015.

30. Caillé S, Sauerwein HC, Schiavetto A, Villemure JG and Lassonde M: Sensory and motor interhemispheric integration after section of different portions of the anterior corpus callosum in nonepileptic patients. Neurosurgery 57: 50-59, 2005. 